\title{
COMBINED NATURAL CONVECTION COOLING OF A DRINK CAN
}

\author{
S. JIRACHEEWANUN ${ }^{\otimes 1,2}$, S. W. ARMFIELD ${ }^{1}$ and M. BEHNIA ${ }^{1}$
}

(Received 31 January, 2010; revised 15 December, 2010)

\begin{abstract}
We investigate natural convection cooling of the fluid in a drink can placed in a refrigerator by simulating the full combined boundary layer system on the can wall. The cylindrical can is filled with water at initial nondimensional temperature 0 , and located within a larger cylindrical container filled with air at initial temperature -1 . The outer container walls are maintained at constant temperature -1 . Initially both fluids are at rest. Two configurations are examined: the first has the inner can placed vertically in the middle of the outer container with no contact with the outer container walls, and the second has the inner can placed vertically at the bottom of the outer container. The results are compared to those obtained by assuming that the inner can walls are maintained at a constant temperature, showing similar basic flow features and scaling relations, but with very different proportionality constants.
\end{abstract}

2000 Mathematics subject classification: primary 80A20.

Keywords and phrases: natural convection cooling, conjugate boundary layer.

\section{Introduction}

The cylindrical container is one of the most common shapes encountered in natural convection heat transfer processes, arising in liquid/gas storage tanks, thermal energy storage systems and food cans, amongst others. The simplest model of natural convection in such a container, vertically oriented along its axis, assumes that the vertical wall is maintained at a uniform temperature, with an adiabatic top and bottom. Past studies using this approach focused predominantly on the case in which the container's contents are heated up by the boundary conditions [1, 2, 4, 5, 13], although recently the case in which the contents are cooled by the boundary conditions has also been investigated by Lin and Armfield [6-8]. This latter case will be considered here.

\footnotetext{
${ }^{1}$ School of Aerospace, Mechanical and Mechatronic Engineering, University of Sydney, New South Wales, Australia; e-mail: sujin.jir@kmutt.ac.th, armfield@aeromech.usyd.edu.au, m.behnia@usyd.edu.au.

${ }^{2}$ Department of Mechanical Technology Education, King Mongkut's University of Technology Thonburi, Bangkok, Thailand.

(C) Australian Mathematical Society 2011, Serial-fee code 1446-1811/2011\$16.00
} 
The evolving process of a transient cooling natural convection flow within a cylindrical can, vertically oriented along its axis and subjected to cooling isothermal boundary conditions, is as follows. Immediately after initiation of the cooling boundary conditions, a natural convection boundary layer develops on the vertical wall. This boundary layer discharges a cooled intrusion at its base, which travels towards the centre of the can bottom. The intrusion rebounds when it reaches the can centre, forming reverse travelling waves that interact with and perturb the boundary layer. The boundary layer continues to feed cooled fluid into the intrusion, which gradually increases in depth. The strong unsteadiness of the intrusion decays during this time, resulting in a quasi-steady state, with stratified cooled fluid at the bottom of the can, gradually increasing in depth as the boundary layer continues to discharge cooled fluid. These effects are similar to those observed in cavities with differential side-wall heating, for example in Patterson and Armfield [10] where a number of scalings were developed to describe the start-up and fully developed flow structures in terms of the control parameters, the Rayleigh number Ra, and Prandtl number Pr.

The simple model described above, in which the can boundary is assumed to be isothermal, may not be an accurate approximation for the case of interest here, that of a drink can placed in a refrigerator. In this case the can has neither isothermal nor isoflux boundary, as the cool air in the refrigerator interacts with the warmer fluid in the can via the combined system consisting of natural convection boundary layers on both the liquid and air sides of the can wall. To better understand this flow, we investigate the combined natural convection cooling of a drink can, simulating the full refrigerator/can system.

\section{Mathematical model}

The case under consideration is the natural convection cooling of the water in a vertical cylindrical can located within a larger vertical cylindrical container of air, with the air initially at a lower temperature than that of the water in the can. The walls of the can are assumed to be very thin, and hence the assumption of zero thermal resistance at the can walls is used, and the heat capacity in the walls is neglected. The walls of the outer container are maintained at a constant temperature $T_{w}$. Initially both fluids are at rest and the temperatures of the air and water are $T_{a}$ and $T_{0}$, respectively. Note that $T_{a}=T_{w}$.

Two configurations are considered for the can. The first, Case 1, has the can located in the middle of the container with no contact between the can and the container walls. The second, Case 2, has the can located at the bottom of the container, with the can bottom in contact with the container bottom; the can bottom is then maintained at temperature $T_{w}$. Schematics for both cases are shown in Figure 1.

2.1. Governing equations The unsteady natural convection flow in the cylindrical container and can is governed by the Navier-Stokes equations and the temperature 

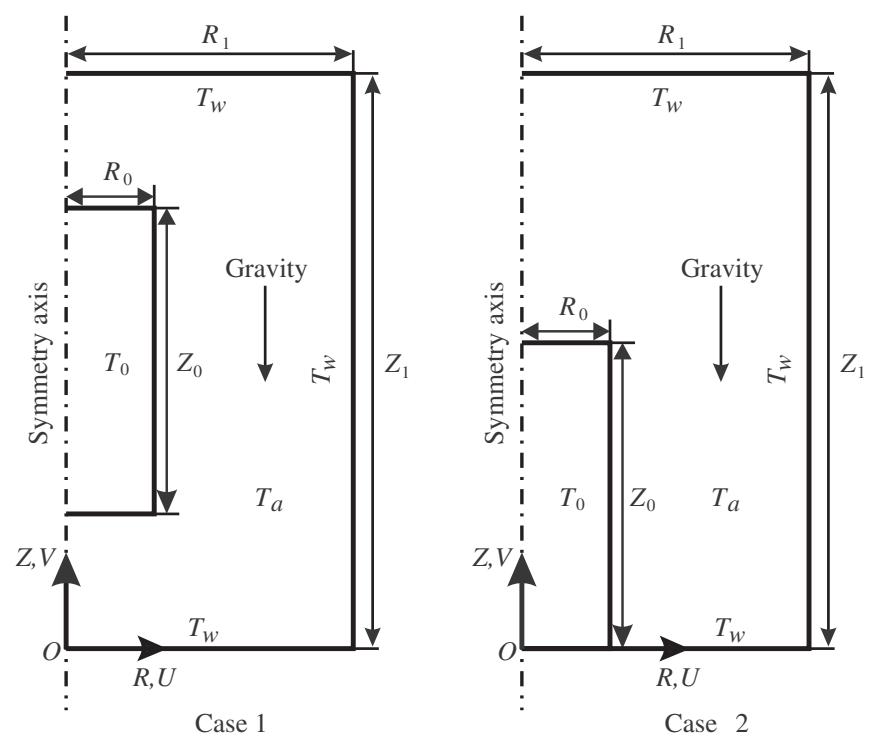

FIGURE 1. Computational domain and coordinate system.

equation. With the Boussinesq approximation, the equations can be written in twodimensional cylindrical coordinates, in nondimensional form, as follows:

$$
\begin{aligned}
\frac{1}{r} \frac{\partial(r u)}{\partial r}+\frac{\partial v}{\partial z} & =0 \\
\frac{\partial u}{\partial \tau}+\frac{1}{r} \frac{\partial(r u u)}{\partial r}+\frac{\partial(v u)}{\partial z} & =-\frac{\partial p}{\partial r}+\frac{\operatorname{Pr}}{\operatorname{Ra}^{1 / 2}}\left[\frac{1}{r} \frac{\partial}{\partial r}\left(r \frac{\partial u}{\partial r}\right)-\frac{u}{r^{2}}+\frac{\partial^{2} u}{\partial z^{2}}\right] \\
\frac{\partial v}{\partial \tau}+\frac{1}{r} \frac{\partial(r u v)}{\partial r}+\frac{\partial(v v)}{\partial z} & =-\frac{\partial p}{\partial r}+\frac{\operatorname{Pr}}{\operatorname{Ra}^{1 / 2}}\left[\frac{1}{r} \frac{\partial}{\partial r}\left(r \frac{\partial v}{\partial r}\right)+\frac{\partial^{2} v}{\partial z^{2}}\right]+\operatorname{Pr} \theta \\
\frac{\partial \theta}{\partial \tau}+\frac{1}{r} \frac{\partial(r u \theta)}{\partial r}+\frac{\partial(v \theta)}{\partial z} & =\frac{1}{\operatorname{Ra}^{1 / 2}}\left[\frac{1}{r} \frac{\partial}{\partial r}\left(r \frac{\partial \theta}{\partial r}\right)+\frac{\partial^{2} \theta}{\partial z^{2}}\right] .
\end{aligned}
$$

The Prandtl number, Pr, and the Rayleigh number, Ra, are defined as

$$
\begin{aligned}
& \operatorname{Pr}=\frac{v}{\alpha}, \\
& \mathrm{Ra}=\frac{g \beta \Delta T Z_{0}^{3}}{\nu \alpha},
\end{aligned}
$$

where $g$ is the acceleration due to gravity; $\beta$ is the coefficient of thermal expansion, $v$ the kinematic viscosity, $\alpha$ the thermal diffusivity, all for water; $\Delta T=T_{a}-T_{0}$ and $Z_{0}$ is the height of the can.

The nondimensional quantities $r, z, u, v, \tau, p$ and $\theta$ are respectively the nondimensional radial coordinate, axial coordinate, $r$-velocity, $z$-velocity, time, 
pressure and temperature, obtained as follows:

$$
\begin{gathered}
r=\frac{R}{Z_{0}}, \quad z=\frac{Z}{Z_{0}}, \quad u=\frac{U}{U_{0}}, \\
v=\frac{V}{U_{0}}, \quad \tau=\frac{t}{Z_{0} / U_{0}}, \quad p=\frac{P}{\rho U_{0}^{2}}, \quad \theta=\frac{T-T_{0}}{T_{0}-T_{w}},
\end{gathered}
$$

where the velocity scale is $U_{0}=\alpha \mathrm{Ra}^{1 / 2} / Z_{0}$, as commonly used for natural convection flow in a differentially side-heated cavity [6-8].

The top, bottom and side boundaries of the container and can are nonslip and impervious, with symmetry boundary conditions applied on $r=0$. The temperature is continuous on the can wall, which has zero thermal resistance and heat capacity. The nondimensional temperature on the container wall is set to $\theta_{w}=-1.0$. Initially both the water and air are quiescent, with the nondimensional water temperature set to $\theta_{0}=0.0$ and the nondimensional air temperature set to $\theta_{a}=-1.0$.

2.2. Numerical method Our simulations were carried out using the commercial solver FLUENT 6.3, in which a finite volume method is used to discretize the governing equations. The equations were integrated in time using the secondorder unsteady segregated solver, with a second-order upwind scheme used for the convective terms in the momentum and energy equations. Second-order central schemes were used for the diffusion and continuity terms. The PREssure STaggering Option (PRESTO) scheme was employed for pressure discretization, with the SIMPLE algorithm used for the pressure-velocity coupling [3, 9, 12].

As most of the flow interaction occurs near the walls, it is necessary to concentrate the mesh points in those regions in both the air and water domains. A nonstaggered mesh, very fine near the walls and relatively coarse in the interior, was used in this study. The results presented below were obtained on a mesh with $111 \times 261$ nodes in the can and, on the boundaries of the container, 181 nodes along the horizontal boundaries and 438 nodes along the vertical boundary. The smallest grid size in the can is $\Delta r=0.000523$, adjacent to the vertical wall; in the container, outside the can, the smallest grid size is $\Delta r=0.000632$, adjacent to the can wall. Results were also obtained on three other meshes with $60 \times 131,45 \times 131$ and $31 \times 69$ nodes in the can, to determine the mesh dependency of the solution.

\section{Results}

According to equation (2.6), the Rayleigh number of a can of water depends on the height of the can and on the physical properties of water. For a can of water $13 \mathrm{~cm}$ high, at $20^{\circ} \mathrm{C}$, placed in a refrigerator containing air at $5{ }^{\circ} \mathrm{C}$, we have $\mathrm{Ra}=4.62 \times 10^{8}$. Here results have been obtained for Rayleigh numbers in the range $\mathrm{Ra}=6.40 \times 10^{7}$ to $6.40 \times 10^{9}$. Owing to the difference in kinematic viscosity, diffusivity and coefficient of thermal expansion for air, the air-side Rayleigh number range is $\mathrm{Ra}_{\text {air }}=5.81 \times 10^{5}$ to $5.81 \times 10^{7}$, again depending on the height of the can. The aspect ratio (height/radius) of the can and container are 4 and 2 , respectively. 


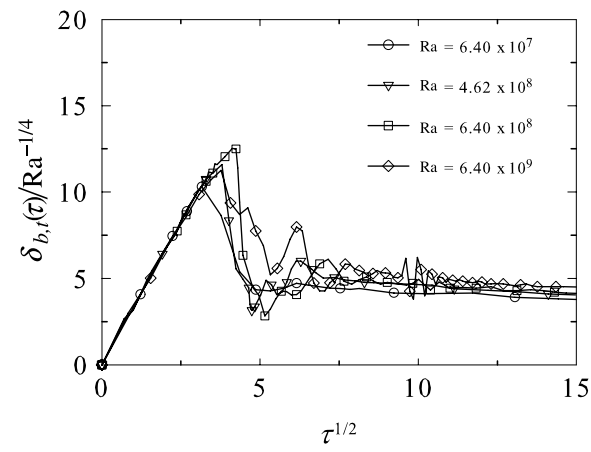

(a) Water domain

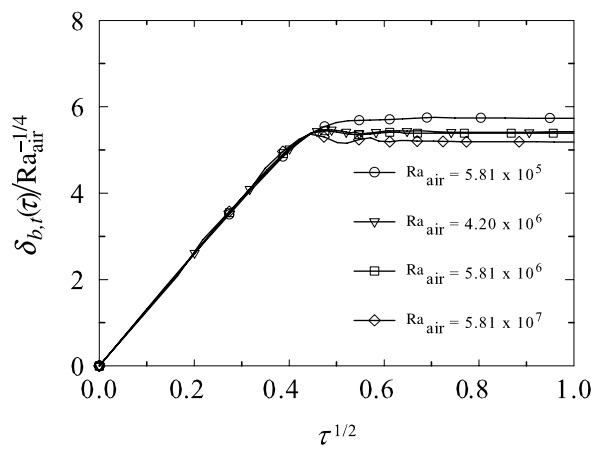

(b) Air domain

FIGURE 2. Scaled thermal boundary layer thickness against scaled time for Case 1 with various Ra.

3.1. Development of the boundary layers Immediately after initiation of the flow, the temperature difference across the can wall leads to the development of natural convection boundary layers on both sides of the wall. The conduction heat transfer through the can wall from the inner (water) side to the outer (air) side results in cooled water immediately adjacent to the wall on the inner side, with an associated descending natural convection boundary layer. On the outer side this results in heated air immediately adjacent to the wall, with an associated ascending natural convection boundary layer. This is qualitatively identical to the process observed by Lin and Armfield [6-8] in their investigation of the natural convection cooling of the fluid in a can subjected to an isothermal wall boundary condition. Following the approach of Patterson and Imberger [11], Lin and Armfield developed a scaling for the thermal boundary layer thickness, $\delta_{b, t}(\tau)$, which was found to obey the relation

$$
\delta_{b, t}(\tau) \sim \tau^{1 / 2} \mathrm{Ra}^{-1 / 4}
$$

Similarly, the dimensionless time $\tau_{b s}$ for the natural convection boundary to reach full development, and the corresponding thermal boundary layer thickness, $\delta_{b s}$, were found to obey the relations

$$
\begin{aligned}
\tau_{b s} & \sim 1, \\
\delta_{b s} & \sim \mathrm{Ra}^{-1 / 4} .
\end{aligned}
$$

Figures 2 and 3 contain the time series of the nondimensional thermal boundary layer thickness, $\delta_{b, t}(\tau)$, on both the air and water sides of the can wall, obtained from the numerical simulations. The thermal boundary layer thicknesses were obtained at half the height of the can wall $\left(Z^{*}=Z_{0} / 2\right.$ where $Z^{*}=0$ at the can bottom), using the location at which the temperature is $\theta=-0.01$ for the water-side boundary layer, and $\theta=-0.99$ for the air-side boundary layer. The boundary layer thickness is scaled by $\mathrm{Ra}^{-1 / 4}$ ( $\mathrm{Ra}_{\text {air }}$ for the air-side boundary layer), and plotted against $\tau^{1 / 2}$ to test the Lin 


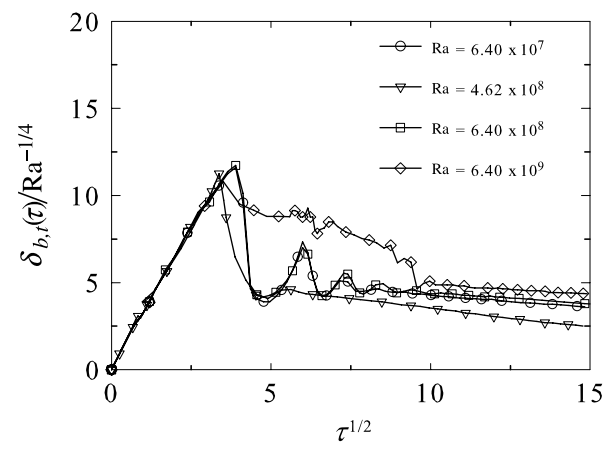

(a) Water domain

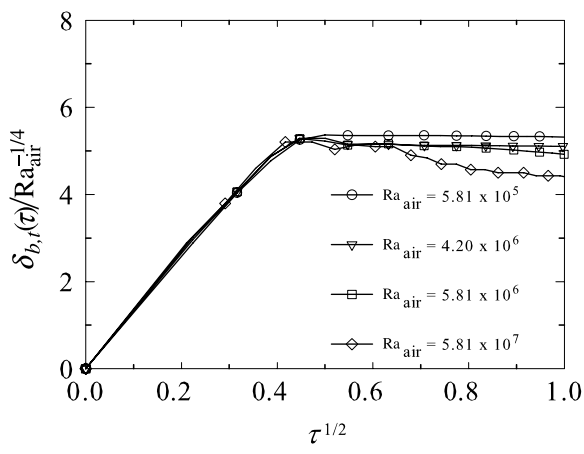

(b) Air domain

FIGURE 3. Scaled thermal boundary layer thickness against scaled time for Case 2 with various Ra.

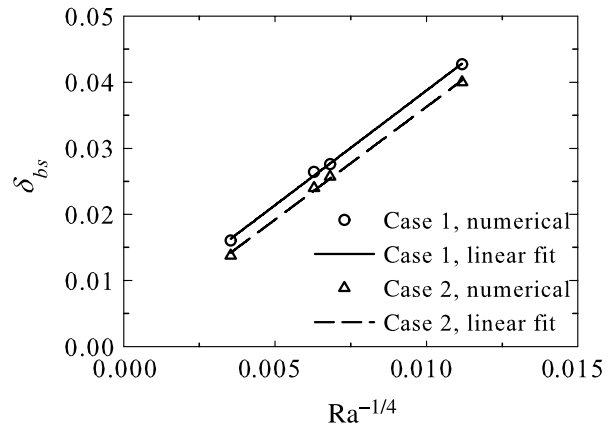

(a) Water domain

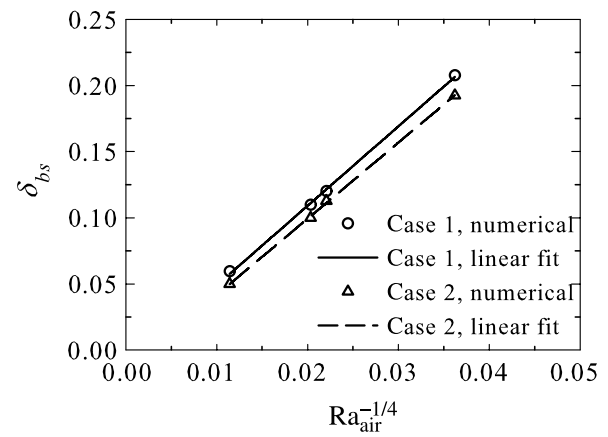

(b) Air domain

FIGURE 4. Fully developed thermal boundary layer thicknesses against $\mathrm{Ra}^{1 / 4}$.

and Armfield scaling given above. The time series are all seen to collapse onto a single straight line for the initial growth of the boundary layer thicknesses on both the water and air sides, for both cases, showing that the scaling works well for this phase of the flow. Overall, the time series show the typical characteristics of growth and transition to full development for natural convection boundary layers. The air-side boundary layer $(\operatorname{Pr}=0.7)$ reaches full development an order of magnitude more rapidly than the water-side boundary layer $(\operatorname{Pr}=7.0)$, transitioning smoothly from growth to full development. The water-side boundary layer grows to a peak that considerably overshoots the fully developed value, as a result of the imbalance in growth rate between the thermal and momentum boundary layers which is characterized by the Prandtl number. The time to full development is seen to be approximately $\tau=5$ for the water-side boundary layer and $\tau=0.5$ for the air-side boundary layer, with little dependency on Rayleigh number, again as predicted by the Lin and Armfield scaling.

Some variation is seen in the scaled fully developed boundary layer thickness, and this is examined further in Figure 4, where the fully developed thermal boundary layer 


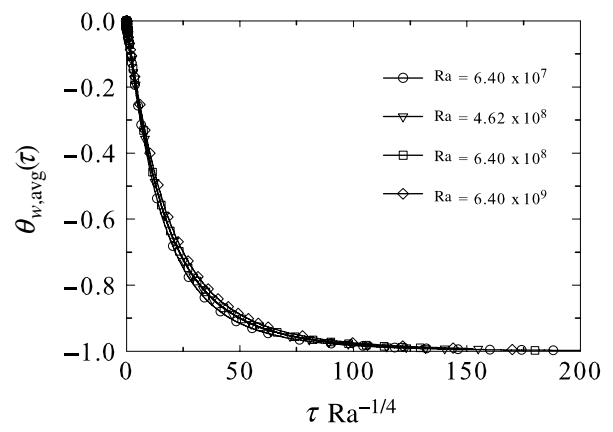

(a) Case 1

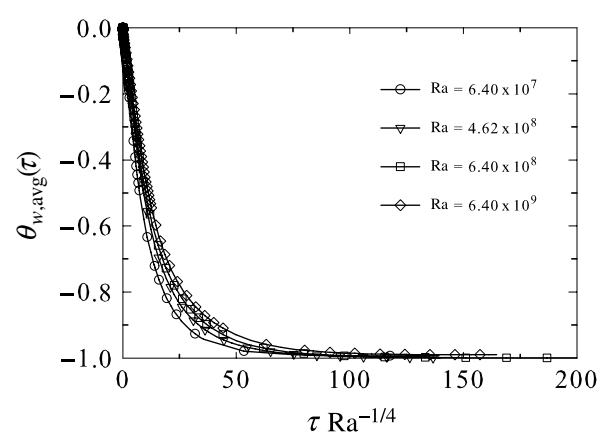

(b) Case 2

FIGURE 5. Average water temperatures against scaled time for various Ra.

thickness at $Z^{*}=Z_{0} / 2$ is plotted against $\mathrm{Ra}^{-1 / 4}$. Again the appropriate Rayleigh number is used. The plots show that $\delta_{b s}$ has a nearly linear relationship with $\mathrm{Ra}^{-1 / 4}$, confirming that the scaling for the thermal boundary layer thickness at full development given in equation (3.3) provides a good prediction of the behaviour in the full combined boundary layer system. It is also observed that the thermal boundary layer thickness varies little between Case 1 and Case 2, with the latter being the smaller.

3.2. Long-term behaviour After the establishment of the thermal boundary layers on both sides of the can wall, the can is gradually filled with cooled fluid, reducing the temperature of the water in the can. The air is initially warmed by the transfer of heat from the water through the can wall, but ultimately that heat is transferred out of the container through the container walls, and at the final steady state the air and water temperatures are equal to -1.0 everywhere. This is again similar to the case considered by Lin and Armfield, who obtained an exponential scaling for the average temperature of the fluid during the long-term phase of the cooling-down process. Written in terms of the Rayleigh number used here, and for a can with a fixed aspect ratio, their scaling is

$$
\theta_{w, \operatorname{avg}}(\tau)=e^{-C_{1} \mathrm{Ra}^{-1 / 4} \tau}-1
$$

where $C_{1}$ is a constant that is determined empirically below. The average temperature of the fluid in the can, $\theta_{w, \text { avg }}$, obtained here as the average of the temperature within the can on the lines $Z^{*}=Z_{0} / 2, r=R_{0} / 2$, is plotted against $\tau \mathrm{Ra}^{-1 / 4}$ in Figures 5(a) and (b) for Case 1 and Case 2, respectively. The time scaling brings all four sets of data to approximately a single curve, indicating that $\tau \mathrm{Ra}^{-1 / 4}$ is an appropriate time scaling for the long-term cooling down of the fluid within the can.

An average value of $C_{1}$ is obtained for each case by fitting an exponential curve to obtain individual values of $C_{1}$ for each $\mathrm{Ra}$, and then averaging. Scaling relations for 
the average temperature of the water in the can for each case are then obtained as

$$
\theta_{w, \text { avg }}(\tau) \simeq e^{-0.051 \mathrm{Ra}^{-1 / 4} \tau}-1
$$

for Case 1, and

$$
\theta_{w, \operatorname{avg}}(\tau) \simeq e^{-0.0716 \mathrm{Ra}^{-1 / 4} \tau}-1
$$

for Case 2.

The constants obtained here, $C_{1}=0.051$ for Case 1 and $C_{1}=0.0716$ for Case 2, may be compared to the corresponding constants obtained by Lin and Armfield [8] for the isothermal boundary configuration. Lin and Armfield also considered two configurations, one with the can wall isothermal and the top and bottom adiabatic, the other with the can wall and bottom isothermal and the top adiabatic. For the first configuration they obtained $C_{1}=5.148$, and for the second $C_{1}=5.428$, where it is noted that the constants they gave in their paper have been appropriately converted to account for the specific time scaling, aspect ratio and definition of Rayleigh number used here. The significantly larger constants obtained by Lin and Armfield show that the rate of cooling is much more rapid for their configurations with isothermal boundary condition.

An important quantity is the total cool-down time, $\tau_{d}$, which is defined to be the time taken for the average temperature of the fluid within the can to reach -0.99 , that is, $99 \%$ of the difference between the boundary condition imposed on the outer container and the initial temperature of the fluid in the can. The total cool-down time

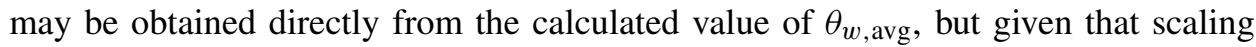

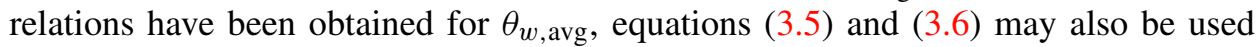
to obtain an estimate for $\tau_{d}$. Using equations (3.5) and (3.6), the cool-down time for $\theta_{w, \text { avg }}$ to reach -0.99 is obtained for Case 1 as

$$
\tau_{d} \simeq 90.297 \mathrm{Ra}^{-1 / 4}
$$

and for Case 2 as

$$
\tau_{d} \simeq 64.300 \mathrm{Ra}^{-1 / 4} .
$$

These cool-down times predicted by the scaling relations are compared to the cooldown times obtained directly from the numerical data, that is, the times at which $\theta_{w, \text { avg }}(\tau)=-0.99$, in Figure 6(a). It is seen that, while providing a reasonable approximation of the cool-down time, the scaling result consistently under-predicts the result obtained directly from the numerical data. Figure 6(b) contains the results obtained from the numerical data plotted against $\mathrm{Ra}^{1 / 4}$, to further test the time scaling. As can be seen, the $\mathrm{Ra}^{1 / 4}$ scaling brings the numerical results very close to the best-fit straight line, providing the empirical relations

$$
\tau_{d} \simeq 149.281 \mathrm{Ra}^{1 / 4}-2874.03
$$

for Case 1, and

$$
\tau_{d} \simeq 117.283 \mathrm{Ra}^{1 / 4}-4959.88
$$

for Case 2. 


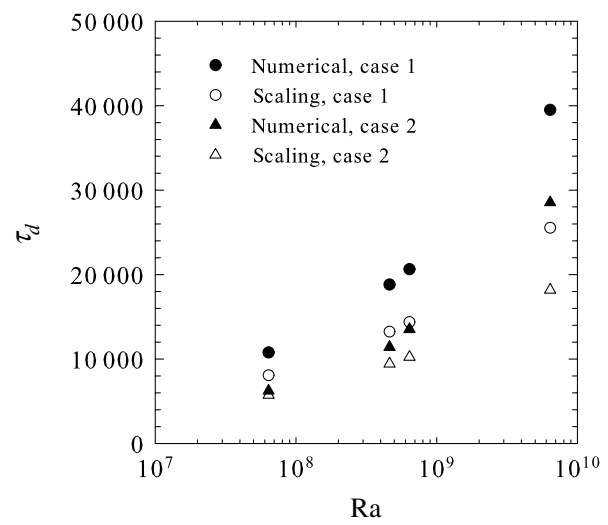

(a)

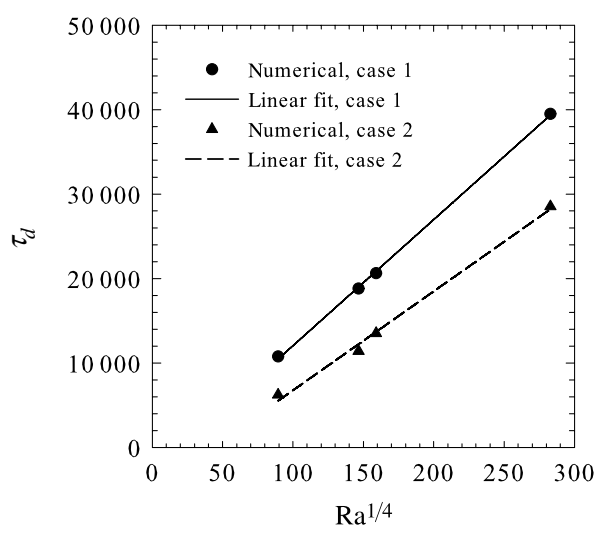

(b)

FIGURE 6. Cool-down time $\tau_{d}$ plotted against Ra, and against $\mathrm{Ra}^{1 / 4}$.

The numerical results, and corresponding scalings given above, show that the Case 2 configuration cools the fluid in the can more rapidly than the Case 1 configuration. For example, for the specific Rayleigh number $\mathrm{Ra}=4.62 \times 10^{8}$, the cool-down time for Case 1 is $\tau_{d}=18817$, while for Case 2 the cool-down time is $\tau_{d}=11414$, a $40 \%$ reduction. By comparison, the cool-down times for the Lin and Armfield [8] isothermal can wall cases, for an equivalent Rayleigh number and aspect ratio, are $\tau_{d}=524$ for their first configuration and $\tau_{d}=552$ for their second configuration. In these cases, the additional application of the isothermal boundary condition on the can bottom had negligible effect on the total cool-down time. It is also apparent that the Lin and Armfield isothermal can wall cases have cool-down times more than an order of magnitude smaller than the cases considered here.

\section{Conclusions}

Full combined boundary layer solutions of the cooling of water in a can within a larger container of cooler air have been obtained, and the results compared to a previous investigation in which the water in the can was cooled via an isothermal can boundary condition. In both cases the basic flow structure within the can is similar, with the development of the natural convection boundary layers adjacent to the can wall obeying the same scaling relations. In particular, the scaling relation for the average temperature of the water is of the same form for the combined boundary layer and isothermal boundary solutions, but with very different scaling constants. The combined boundary layer solutions have total cool-down times more than an order of magnitude greater than the isothermal boundary solutions, showing that the isothermal boundary condition does not provide a good approximation of the cooling time for the combined boundary layer solution. It is also observed that Case 2 considered here, in which the can bottom is in contact with the container bottom, has a $40 \%$ reduction in cool-down time as compared to that of Case 1. 


\section{References}

[1] A. K. Datta and A. A. Teixeira, "Numerically predicted transient temperature and velocity profiles during natural convection heating of canned liquid food", J. Food Sci. 53 (1988) 191-195.

[2] L. B. Evans, R. C. Reid and E. M. Drake, "Transient natural convection in a vertical cylinder", AIChE J. 14 (1968) 251-259.

[3] A. Gustavsen, B. T. Griffith and D. Arasteh, "Natural convection effects in three-dimensional window frames with internal cavities", ASHRAE Trans. 107 (2001) 527-537.

[4] J. M. Hyun, "Transient process of thermally stratifying an initially homogeneous fluid in an enclosure”, Int. J. Heat Mass Transfer 27 (1984) 1936-1938.

[5] A. Kumar and M. Bhattacharya, "Transient temperature and velocity profiles in a canned nonNewtonian liquid food during sterilization in a still-cook retort", Int. J. Heat Mass Transfer 34 (1991) 1083-1096.

[6] W. Lin and S. W. Armfield, "Direct simulation of natural convection cooling in a vertical circular cylinder", Int. J. Heat Mass Transfer 42 (1999) 4117-4130.

[7] W. Lin and S. W. Armfield, "Natural convection cooling of rectangular and cylindrical containers", Int. J. Heat Mass Transfer 22 (2001) 72-81.

[8] W. Lin and S. W. Armfield, "Long-term behavior of cooling fluid in a vertical cylinder", Int. J. Heat Mass Transfer 48 (2005) 53-66.

[9] S. V. Patankar, Numerical heat transfer and fluid flow (Hemisphere, Washington, DC, 1980).

[10] J. C. Patterson and S. W. Armfield, "Transient features of natural convection in a cavity", J. Fluid Mech. 219 (1990) 469-497.

[11] J. C. Patterson and J. Imberger, "Unsteady natural convection in a rectangular cavity", J. Fluid Mech. 100 (1980) 65-86.

[12] R. Peyret, Handbook of computational fluid mechanics (Academic Press, London, 1996).

[13] V. I. Polezhaev and S. G. Cherkasov, "Unsteady thermal convection in a cylindrical vessel heated from the side", Fluid Dyn. 18 (1983) 620-629. 